\title{
Avaliação do impacto da exposição a agrotóxicos sobre a saúde de população rural. Vale do Taquari (RS, Brasil)
}

\author{
Evaluation of the impact of exposure to pesticides \\ on the health of the rural population. \\ Vale do Taquari, State of Rio Grande do Sul (Brazil)
}

\author{
Andressa de Souza ${ }^{1}$ \\ Afonso dos Reis M edeiros ${ }^{1}$ \\ Ana Cláudia de Souza ${ }^{1}$ \\ Márcia Wink ${ }^{2}$ \\ Ionara Rodrigues Siqueira ${ }^{3}$ \\ M aria Beatriz Cardoso Ferreira ${ }^{3}$ \\ Luciana Fernandes ${ }^{1}$ \\ M aria Paz Loayza Hidalgo ${ }^{4}$ \\ Iraci Lucena da Silva Torres ${ }^{3}$
}

${ }^{1}$ Centro Universitário

Univates.

${ }^{2}$ Universidade Federal de

Ciências M édicas de Porto

Alegre.

${ }^{3}$ Instituto deCiências

Básicas da Saúde,

Departamento de

Farmacologia, Universidade

Federal do Rio Grande do

Sul. Rua Sarmento Leite

500/202. 90050-170 Porto Alegre RS.

iracitorres@gmail.com

${ }^{4}$ Departamento de

Psiquiatria, U niversidade

Federal do Rio Grandedo

Sul.
Abstract The scope of this cross-sectional study was to assess a possible link between contact with pesticides and the prevalence of chronic disease in the rural population in the south of Brazil. Three municipalities were randomly chosen. The sample selected was recruited over three months (2005) and was composed of 298 subjects of both sexes, between 18 and 65 years of age, who fre quented public or private pharmacies for the purchase of medication. Data obtained by means of structured intervi ews revealed that $68.4 \%$ of those interviewed worked in rural activities, and $74.8 \%$ of these were members of families working in agriculture with contact with pesticides. The average age was $51 \pm 16.5$ years of age for the interviewees with contact with pesticides and $50 \pm 17.9$ years for those without contact. The sample was composed of $36.2 \%$ of men, and $57.7 \%$ of the individuals had more than four years of education. Direct or indirect contact with pesticides was associated with the report of several diseases, with neurol ogical and oral diseases being the most prevalent. There was a link with painful conditions and individuals with contact with pesticides re ported twice as many diseases. The data corroborate the literature and draw attention to the need for promoting measures to protect the health of the rural population.

Key words Pesticides, Agricultural workers and health
Resumo Este estudo transversal teve o objetivo de avaliar possível associação entrecontato com agrotóxicos e prevalência de doenças crônicas em população rural do Sul do Brasil. Três municípios foram aleatoriamente escolhidos. A amostra selecionada por conveniência foi recrutada durante três meses (2005) e se compôs de 298 sujeitos de ambos os sexos, com idade entre 18 e 65 anos eque procuravam farmácia pública ou privada para a compra de medicamentos. Os dados obtidos, me dianteentrevistas estruturadas, demonstraram que $68,4 \%$ dos entrevistados exerciam atividade rural, dosquais $74,8 \%$ eram membros defamílias deagricultores e tinham contato com agrotóxicos. A mé dia deidadefoi $51 \pm 16,5$ anos para os entrevistados com contato com agrotóxicos e $50 \pm 17,9$ anos para os sem contato. A amostra foi constituída de $36,2 \%$ por homens e $57,7 \%$ dos indivíduos que apresentavam mais de quatro anos de estudo. 0 contato direto ou indireto com agrotóxicos associou-se ao relato de várias doenças, sendo as neurológicas eas orais as mais prevalentes. Houve associação com relato de condições dol orosas, de modo que indivíduos com contato com agrotóxicos apresentaram em torno de duas vezes mais chances de as referirem. Os dados corroboram os da literatura eindicam a necessidade de promoção de medidas deproteção e prevenção da saúde da população rural. Palavras-chave Agrotóxicos, Trabalhadores agrícolas e saúde 
Introdução

O Brasil é o terceiro mercado e o oitavo maior consumidor deagrotóxicos por hectare do mundo, sendo herbicidas e inseticidas responsáveis por $60 \%$ dos produtos comercializados no país ${ }^{1,2}$. Há relato de que a utilização indiscriminada de agrotóxicos no meio rural pode provocar intoxicação de seus trabalhadores com diferentes graus de severidade e levar à depressão e, até mesmo, ao suicídio ${ }^{3}$. Os agrotóxicos atuam diretamente, por meio do contato direto do organismo com essas substâncias, ou indiretamente, por intermédio do desenvolvimento de algum fator impactante como resultado do uso desses agentes químicos ${ }^{4}$. Três são as principais vias responsáveis pelo impacto direto da contaminação humana: a ocupacional, que se caracteriza pela contaminação dos trabalhadores que manipuIam essas substâncias; a ambiental, que ocorre por meio de dispersão/distribuição dos agrotóxicos ao longo dos diversos componentes do meio ambiente; e a alimentar que se dá pela contaminação relacionada à ingestão de produtos contaminados por agrotóxicos4.

Intoxicações por agrotóxicos podem provocar diminuição das defesas imunológicas, anemia, impotência sexual, cefaleia, insônia, alterações de pressão arterial, distimias e distúrbios de comportamento. Estas manifestações são frequentes entre os agricultores, determinando, por vezes, a proibição médica do trabalho na lavoura ea orientação para outro tipo de atividade profissional ${ }^{5}$.

Os agrotóxicos tiveram seu uso fortemente estimulado por políticas de estado, a partir da década de 1970, incluindo a concessão de crédito agrícola, naquele período, vinculado à sua aquisição, e pela oferta comercial queexaltava suas propriedades de reduzir o trabal ho com pragas e de beneficiar alimentos, população e trabalhadores. Assim, passaram os agrotóxicos a estar presente na vida diária de milhões de trabalhadores do campo, que a eles se expõem ocupacionalmente com suas famílias. Paralelamente, também se incorporaram à dieta dos brasileiros do campo e das cidades, presentes que estão nos al imentos.

0 Vale do Taquari é uma região localizada no extremo sul do Brasil, composta de 37 municípios, nos quais se destaca o setor de agropecuária. Está situado na região central do Rio Grande do Sul, e sua população tem em torno de 320 mil habitantes, o que equivale a 2,97\% da população gaúcha, sendo a grande maioria, descendentes de alemães e, em menor proporção, de italianos e em uma pequena parte de açorianos. 0 aparecimento da indústria e o desenvolvimento de comércio e setor de serviços provocaram mudanças significativas nosúltimos 30 anos. A expectativa devida aumentou, sendo, no momento, de 73,6 anos. Segundo dados do IBGE referentes ao ano de 2000, o índice de alfabetização na região é de 93,27\%. O PIB per capita de 2004 foi de $R \$ 17.002,00$.

Infelizmente, são escassos os estudos de base populacional sobre as características da utilização ocupacional ou sobre as intoxicações por agrotóxicos. Cabe ressaltar que o uso indevido de pesticidas contribui intensamente para a degradação ambiental, além de ser frequentea ocorrência de intoxicações ocupacionais, constituindo um dos principais problemas de saúde pública no meio rural brasileiro.

Este estudo teve por objetivo avaliar a associação entre contato com agrotóxicos e prevalência de doenças crônicas, abrangendo a população do Vale do Taquari que compareceu a estabelecimentos de dispensação de medicamentos de setores público e privado no período dejulho a setembro de 2005.

\section{Material e métodos}

Trata-se de um estudo com delineamento transversal. Foram escolhidos, deforma al eatória, três municípios dentro os 37 que compõem o Valedo Taquari, importante zona agrícola do Rio Grande do Sul. Fez-seum sorteio utilizando o programa Epi-Info para evitar o viés de amostragem, como, por exemplo, haver maior prevalência de alguma doença, ou por escolher um município que apresentasse melhores condições de saúde. Essa região foi escolhida devido à intensa utilização de agrotóxicos nas lavouras. Os três municípios selecionados são Travesseiro (2.276 habitantes), Dr.Ricardo (2.272 habitantes) e Westfália (2.744 habitantes), cujas economias baseiamse em atividades rurais (agricultura ou pecuária). Todos os municípios indispunham de apenas uma drogaria e de uma farmácia pública (em Posto deSaúde M unicipal). A amostra selecionada foi de conveniência recrutada em farmácias. Durante um período de três meses (julho a setembro de 2005), todos os sujeitos, entre 18 e 65 anos, que buscavam a farmácia para a compra de medicamentos ou para a busca de orientação farmacológica foram entrevistados. A farmácia foi considerada como um lugar que concentrava a população que busca auxílio para sua saúde e, portanto com maior probabilidade de apresentar algum dos desfechos de interesse. 
O levantamento de dados foi feito por meio de entrevistas estruturadas. Os avaliadores eram estudantes de Iniciação Científica dos cursos de graduação em Farmácia do Centro Universitário Univates de Lajeado (RS), previamente treinados. 0 questionário utilizado na entrevista foi elaborado para avaliar os seguintes parâmetros demográficos e comportamentais: município, gênero, idade (em anos completos), escolaridade (em anos de estudo), ocupação, região em que habitava (rural versus urbana), tabagismo, consumo deálcool equalidadedesono. Também se avaliou o relato de doenças ou manifestações relacionadas, incluindo hipertensão arterial sistêmica, hipercolesterolemia, hiperglicemia, artrite, cefaleia, doenças neurológicas, ansiedade, depressão, transtornos do sono, câncer, hérnia, doenças respiratórias, renais, cardíacas, orais, cutâneas, oculares, vasculares, gastrintestinais e endócrinas. Analisaram-se a prevalência de relato dessas doenças e manifestações específicas e a possível associação com contato com agrotóxicos.

Adicionalmente, doenças e fatores de risco relacionados ao sistema cardiovascular foram agrupados (incluindo hipertensão arterial sistê mica, doenças vasculares, cardíacas e renais, hiperglicemia, hipercolesterolemia, uso detabaco e consumo de bebidas alcoólicas), e os indivíduos da amostra foram categorizados entre aqueles que relatavam pelo menos uma doença ou um fator de risco e os que não apresentavam qualquer doença ou fator de risco cardiovascular. $M$ anifestações relacionadas ao sistema nervoso central também foram agrupadas em condições dolorosas (cefaleia, artrite e doenças neurológicas) epsiquiátricas (ansiedade, depressão etranstornos do sono). Novamente criaram-se duas categorias, compostas pelosindivíduos que apresentavam pelo menos uma manifestação ou ne nhuma manifestação dolorosa ou psiquiátrica.

Os grupos foram divididos considerando-se o fator em estudo - contato com agrotóxicos (contato direto ou indireto). Foram considerados desfechos primários relatos de artrite, doenças neurológicas, doenças psiquiátrica, cardiovascular, gastrintestinal, câncer, cefaleia, doenças respiratórias, orais, cutâneas, oculares e endócrinas.

A amostra foi calculada no programa EpiInfo, considerando nível de confiança de $95 \%$, poder estatístico de $80 \%$ eprevalência mínima de morbidade para os sem contato de $7 \%$. Foram entrevistados 298 indivíduos que buscavam medicamentos nos 6 estabelecimentos sel ecionados, sendo cerca de 100 entrevistas em cada município.
Variáveis contínuas foram analisadas pelo teste t de Student para amostras independentes. Análise univariada e teste qui-quadrado $\left(c^{2}\right)$ ( $M$ antel-H aensel com correção de Yates) ou teste exato de Fisher foram usados para verificar diferenças em proporções para as variáveis estudadas. A análise de regressão logística foi utilizada para avaliar variáveis de confusão everificar a existência de associação entre contato com agrotóxico e relato de doença ou sintomatologia específica. Variáveis que mostraram significativa associação foram incluídas em regressão logística multivariada (enter procedure). Valores de $P$ menores que 0,20 foram requeridos para o fator ser incluído na análise. Os valores foram considerados significativos quando o erro de tipo I (Pá) foi menor ou igual a 0,05.

Simultaneamente às coletas, os questionários foram revisados e codificados. Foi realizada dupla digitação cruzada, gerando banco de dados no Programa Epi-Info. A análise estatística foi realizada utilizando-se o programa SPSS for Windows, versão 10.0 .

0 protocolo segue as condições estabelecidas na Resolução 196/96 do Conselho Nacional de Saúde (CNS) efoi aprovado pelo Comitê de Ética da Universidade do Vale do Rio dos Sinos. Todos os participantes que concordaram em integrar-se ao estudo assinaram o Termo de Consentimento LivreeEsclarecido.

\section{Resultados}

Perfil sociodemográfico dos entrevistados

No total da amostra ( $n=298$ ), $68,4 \%$ informaram utilizar de agrotóxicos. Não houve diferença significativa entre os municípios quanto a esse uso (teste qui-quadrado; $\mathrm{P}=0,06$ ), observando-se taxas de $71 \%, 75 \%$ e $60 \%$, respectivamente, em Travesseiro, Dr. Ricardo e Westfália. A maior parte da amostra foi composta por muIheres $(63,8 \%)$, sendo que $61,6 \%$ delas relataram ter contato com agrotóxicos. Observou-se associação significativa entregênero eessetipo de contato (teste exato de Fisher, com resíduos ajustados; $P=0,001$ ). 0 número de mulheres sem contato com agrotóxicos foi significativamente maior, com razão de chances de 0,39 (IC95\%: 0,220,68) (Tabela 1).

Não houve diferença significativa entre os grupos com e sem contato com agrotóxico na variável idade $(51 \pm 16,5$ anos e $50 \pm 17,9$ anos respectivamente, teste $t$ de Student para amostras 
Tabela 1. Características da amostra estudada.

\begin{tabular}{|c|c|c|c|c|c|c|}
\hline \multirow[b]{2}{*}{ Características } & \multirow[b]{2}{*}{ Frequências } & \multicolumn{2}{|c|}{ Contato com agrotóxicos } & \multirow[b]{2}{*}{$\mathrm{p}$} & \multirow{2}{*}{$\begin{array}{l}\text { Razão de } \\
\text { chances }\end{array}$} & \multirow[b]{2}{*}{ IC95\% } \\
\hline & & $\operatorname{Sim}(n=204)$ & Não (n=94) & & & \\
\hline Gênero ${ }^{a}$ & & & & 0,001 & 0,39 & $0,22-0,68$ \\
\hline Feminino & $190(63,8 \%)$ & $117(57,4 \%)$ & $73(77,7 \%)$ & & & \\
\hline Masculino & $108(36,2 \%)$ & $87(42,6 \%)$ & $21(22,3 \%)$ & & & \\
\hline Idade (média $\pm \mathrm{DP})^{\mathrm{b}}$ & - & $51 \pm 16,5$ & $50 \pm 17,9$ & 0,680 & - & - \\
\hline Ocupação a & & & & 0,000 & 7,61 & $4,41-13,14$ \\
\hline Rural & $192(64,4 \%)$ & $161(78,9 \%)$ & $31(33,0 \%)$ & & & \\
\hline Urbana ou do lar & $106(35,6 \%)$ & $43(21,1 \%)$ & $63(67,0 \%)$ & & & \\
\hline Escolaridade (anos de estudo) a & & & & 0,060 & 1,66 & $0,99-2,75$ \\
\hline Menos de 4 & $126(42,3 \%)$ & $94(46 \%)$ & $32(34 \%)$ & & & \\
\hline Igual ou mais de 4 & $172(57,7 \%)$ & $110(54 \%)$ & $62(66 \%)$ & & & \\
\hline Região em que vive ${ }^{a}$ & & & & 0,000 & 8,90 & $4,94-16,02$ \\
\hline Rural & $223(74,8 \%)$ & $180(88,2 \%)$ & $43(45,7 \%)$ & & & \\
\hline Urbana & $75(25,2 \%)$ & $24(11,8 \%)$ & $51(54,3 \%)$ & & & \\
\hline Hábito de fumar a & & & & 0,854 & 0,91 & $0,45-1,86$ \\
\hline Sim & $39(13,1 \%)$ & $26(12,7 \%)$ & $13(13,8 \%)$ & & & \\
\hline Não & $259(86,9 \%)$ & $178(87,3 \%)$ & $81(86,2 \%)$ & & & \\
\hline Consumo de álcool a & & & & 0,046 & 1,66 & $1,02-2,72$ \\
\hline Sim & $162(54,4 \%)$ & $119(58,3 \%)$ & $43(45,7 \%)$ & & & \\
\hline Não & $136(45,6 \%)$ & $85(41,7 \%)$ & $51(54,3 \%)$ & & & \\
\hline Padrão de sono ${ }^{a}$ & & & & 1,000 & 1,01 & $0,61-1,69$ \\
\hline Dorme bem & $194(65,1 \%)$ & $133(65,2 \%)$ & $61(64,9 \%)$ & & & \\
\hline Razoável ou dorme mal & $104(34,9 \%)$ & $71(34,8 \%)$ & $33(35,1 \%)$ & & & \\
\hline
\end{tabular}

${ }^{a}$ Comparação entregrupos realizada por meio detestequi-quadrado ou testeexato deFisher. ${ }^{b} \mathrm{C}$ omparação entregrupos realizada por meio detestet deStudent para amostras independentes.

independentes; $P>0,05)$. Considerando-se a amostra total entrevistada, $42,3 \%$ apresentaram grau de escolaridade inferior a 4 anos de estudo, não havendo diferença significativa entre os grupos com e sem contato com agrotóxicos (teste exato de Fisher; $\mathrm{P}=0,06)$ (Tabela 1 ).

Dentre os entrevistados, $64,4 \%$ exerciam atividades rurais. Destes, aproximadamente $84 \%$ (161 indivíduos em um total de 192) tinham contato com agrotóxicos. O bservou-se associação significativa entre a ocupação do entrevistado e esse tipo de contato (teste exato de Fisher, com resíduos ajustados; $P=0,000$ ). Indivíduos que exerciam atividades rurais mostraram significativamente maior contato com agrotóxicos, com razão de chances de 7,61 (IC95\%: 4,41-13,14). Padrão similar foi observado em relação à re gião onde o entrevistado vivia e ao contato com agrotóxicos (teste exato de Fisher, com resíduos ajustados; $P=0,000$ ). Indivíduos vivendo em região rural mostraram significativamente maior contato com agrotóxicos, com razão de chances de 8,9 (IC95\%: 4,94-16,02) (Tabela 1).

Considerando-se o hábito de fumar, 13,1\% relataram ser fumantes, não havendo diferença significativa entre os indivíduos com e sem-contato com agrotóxicos (teste exato de Fisher; $P=0,85)$. Por outro lado, $54,4 \%$ dos entrevistados relataram consumo de álcool, havendo associação significativa com contato com agrotóxicos (teste exato de Fisher, com resíduos ajustados; $P=0,046$ ). Observou-se que indivíduos com contato com agrotóxicos têm aproximadamente duas vezes mais chance de consumir bebidas alcoólicas (razão de chances: 1,66; IC95\%: 1,022,72) (Tabela 1).

Com relação à qualidade do sono, $65,1 \%$ relataram que dormiam bem. Destes, $68,6 \%$ tinham contato com agrotóxicos, não havendo diferença significativa em relação aos indivíduos sem contato (teste exato deFisher; $P=1,0$ ) (Tabela 1). $\mathrm{Na}$ amostra total, 91,5\% relataram dormir 6 horas ou mais por noite.

Perfil de saúde da população

$\mathrm{Na}$ amostra estudada, 86,9\% dos entrevistados relataram apresentar pelo menos uma doença. Entre os indivíduos com contato com agrotóxicos, esse relato ocorreu em $89,2 \%$ dos casos, 
enquanto entre os sem contato a ocorrência foi de $82 \%$. N ão foi observada associação entre relato de doença e contato com agrotóxicos (teste exato de Fisher, com resíduos ajustados; $\mathrm{P}=0,10$ ). No entanto, ao se avaliar o número de doenças relatadas pelos entrevistados, observaram-se diferen ças significativas entreindivíduos com esem contato (teste t de Student para amostras independentes; $P=0,001)$, com valores médios ( $\pm D P$ ), respectivamente, de $2,48( \pm 0,95)$ e $2,12( \pm 0,77)$.

Por outro lado, houve significativa associação entre relato de doenças na família e contato com agrotóxicos (teste exato de Fisher, com resíduos ajustados; $\mathrm{P}=0,018$ ). Observou-se que $70,1 \%$ dos indivíduos com contato relataram doenças na família, enquanto essa frequência foi de $55,3 \%$ entre os sem contato, com uma razão dechances de 1,89 (IC95\%: 1,14-3,14). Ao se avaliar o número de doenças na família relatadas pelos entrevistados, também foram verificadas diferenças significativas entreindivíduos com esem contato com agrotóxicos (testet de Student para amostras independentes; $P=0,008)$, com valores médios $( \pm D P)$, respectivamente, de $1,12( \pm 1,04)$ e $0,80( \pm 0,84)$.

Ao se avaliarem as doenças e as manifestações específicas relatadas pel os entrevistados, por meio de análise univariada, observou-se associação significativa apenas entre contato com agrotóxicos e relato de doenças orais ou neurológicas (teste exato de Fisher, com resíduos ajustados; respectivamente, $\mathrm{P}=0,02, \mathrm{P}=0,01$ ) (Tabela 2). Quanto às doenças orais, nenhum dos indivíduos sem contato com agrotóxicos relatou a sua presença, enquanto $3,7 \%$ dos com contato referiram tê-las. Quanto ao relato de doenças neurológicas, indivíduos com contato com agrotóxicos apresentaram 2,5 vezes mais chances de as referirem do que os sem contato.

Ao ser feito controle para colinearidadeentre as variáveis, por meio da análise de regressão logística, constatou-se que apenas a artrite e as doenças neurológicas foram mantidas no modelo $(\mathrm{P}<0,05)$.

Agrupando-se doenças e manifestações específicas relatadas pelos pacientes (Tabela 3), não se registrou associação entre relato de doenças cardiovasculares ou psiquiátricas com contato com agrotóxicos (teste qui-quadrado ou teste exato de Fisher; $P>0,05$ ). Por outro lado, houve associação entre o relato de condições dolorosas e esse tipo de contato (teste exato de Fisher; $P=0,02$ ), de modo que indivíduos com contato com agrotóxicos apresentaram aproximadamente 2 vezes mais chances de as referirem do que os sem contato (Tabela 3).

\section{Discussão}

A amostra ora analisada apresentou média de idade em torno de 50 anos. Do total da amostra $68,4 \%$ relataram ter contato com agrotóxicos, $64,4 \%$ exerciam atividades ruraise, destes, $78,9 \%$ estavam em contato com agrotóxicos. A maior parte da amostra foi composta por mulheres (63,8\%), o que não surpreende, pois, nesse tipo de comunidade, são geralmente elas que procuram tanto os estabelecimentos de dispensação de medicamentos, como os de comércio em geral.

Ao analisaram-se os dados considerando 0 gênero, verificou-sequehomenstêm maior contato com agrotóxicos, confirmando dados obtidos em estudo realizado na região de Nova Friburgo, Rio deJ aneiro ${ }^{4}$, em que se observou queo trabalho rural era realizado majoritariamente por homens. Por outro lado, estudo desenvolvido na Serra Gaúcha, utilizando delineamento transversal e que avaliou o uso de agrotóxicos, não encontrou diferenças por gêner $0^{6}$.

Os indivíduos com contato com agrotóxicos relataram maior consumo de bebidas alcoólicas em relação aos sem contato. Existem interessantes similaridades entre os efeitos à saúde provocados por sobre-exposição a pesticidas e o consumo de álcool. Ambos podem causar distúrbios aos sistemas nervoso central e periférico com características semel hantes. A encefal opatia tóxica crônica devido a pesticidas é caracterizada por déficit neurocomportamental similar àqueleque é observado após ingestão alcoólica crônica, tais como distúrbio neuropsicomotor e déficit da memória de curta duração. 0 alcoolismo é um fator confundidor no diagnóstico diferencial com a intoxicação crônica induzida por pesticidas, seja pelos quadros de polineuropatia seja pelos quadros de síndrome de abstinência alcoólica ${ }^{7,8}$. 0 álcool pode também causar disfunção hepática e alterações nos testes de inibição das colinesterases. Estudo realizado em Nova Friburgo ${ }^{9}$ destaca a el evada prevalência de alcoolismo em grupo de agricultores. Sintomas de abstinência alcoólica também levam a quadros variados de sintomas neurocomportamentais e neurológicos periféricos. Está bem estabelecido que o álcool apresenta ação mutagênica, carcinogênica eteratogênica ${ }^{10}$. As bebidas alcoólicas são misturas complexas de diferentes tipos de álcoois, aldeídos e ésteres, além de vários compostos orgânicos e inorgânicos. A ativação de metabólitos, a interferência nos mecanismos de reparo de DNA ou ainda a exacerbação da deficiência nutricional são mecanismos pelos quais o álcool pode aumentar 


\begin{tabular}{|c|c|c|c|c|c|c|}
\hline \multirow{2}{*}{$\begin{array}{c}\text { Doenças e manifestações } \\
\text { específicas relatadas pelo } \\
\text { indivíduo }\end{array}$} & \multirow[b]{2}{*}{ Frequências } & \multicolumn{2}{|c|}{ Contato com agrotóxicos } & \multirow[b]{2}{*}{$\mathrm{p}$} & \multirow{2}{*}{$\begin{array}{l}\text { Razão de } \\
\text { chances }\end{array}$} & \multirow[b]{2}{*}{ IC95\% } \\
\hline & & $\operatorname{Sim}(n=204)$ & Não (n=94) & & & \\
\hline \multicolumn{4}{|l|}{ Hiperglicemia } & \multirow[t]{3}{*}{0,53} & \multirow[t]{3}{*}{1,43} & \multirow[t]{3}{*}{$0,58-3,48$} \\
\hline Sim & $28(9,4 \%)$ & $21(10,3 \%)$ & $7(7,4 \%)$ & & & \\
\hline Não & $270(90,6 \%)$ & $183(89,7 \%)$ & $87(92,6 \%)$ & & & \\
\hline \multicolumn{4}{|l|}{ Hipercolesterolemia } & \multirow[t]{3}{*}{0,48} & \multirow[t]{3}{*}{1,35} & \multirow[t]{3}{*}{$0,65-2,82$} \\
\hline Sim & $42(14,1 \%)$ & $31(15,2 \%)$ & $11(11,7 \%)$ & & & \\
\hline Não & $256(85,9 \%)$ & $173(84,8 \%)$ & $83(88,3 \%)$ & & & \\
\hline \multicolumn{4}{|l|}{ Cefaleia } & \multirow[t]{3}{*}{0,12} & 1,61 & $0,89-2,89$ \\
\hline Sim & $78(26,2 \%)$ & $59(-28,9 \%)$ & $19(20,2 \%)$ & & & \\
\hline Não & $220(73,8 \%)$ & $145(71,1 \%)$ & $75(79,8 \%)$ & & & \\
\hline Distúrbios do sono & & & & 1,00 & 0,99 & $0,59-1,65$ \\
\hline Sim & $104(34,9 \%)$ & $71(34,8 \%)$ & $33(35,1 \%)$ & & & \\
\hline Não & $194(65,1 \%)$ & $133(65,2 \%)$ & $61(64,9 \%)$ & & & \\
\hline Depressão & & & & & & \\
\hline Sim & $80(26,8 \%)$ & $57(27,9 \%)$ & $23(24,5 \%)$ & 0,58 & 1,20 & $0,68-2,10$ \\
\hline Não & $218(73,2 \%)$ & $147(72,1 \%)$ & $71(75,5 \%)$ & & & \\
\hline Ansiedade & & & & 0,58 & 1,21 & $0,70-2,08$ \\
\hline $\mathrm{Sim}$ & $87(29,2 \%)$ & $62(30,4 \%)$ & $25(26,6 \%)$ & & & \\
\hline Não & $211(70,8 \%)$ & $142(69,6 \%)$ & $69(73,4 \%)$ & & & \\
\hline Doenças N eurológicas & & & & $0,01^{*}$ & 2,52 & $1,17-5,42$ \\
\hline $\operatorname{Sim}$ & $52(17,5 \%)$ & $43(21,1 \%)$ & $9(9,6 \%)$ & & & \\
\hline Não & $246(82,5 \%)$ & $161(78,9 \%)$ & $85(90,4 \%)$ & & & \\
\hline Artrite & & & & 0,09 & 3.12 & $0,90-10,80$ \\
\hline Sim & $22(7,4 \%)$ & $19(9,3 \%)$ & $3(3,2 \%)$ & & & \\
\hline Não & $276(92,6 \%)$ & $185(90,7 \%)$ & $91(96,8 \%)$ & & & \\
\hline Câncer & & & & 0,06 & 1,48 & $1,37-1,60$ \\
\hline Sim & $8(2,7 \%)$ & $8(3,9 \%)$ & 0 & & & \\
\hline Não & $290(97,3 \%)$ & $196(96,1 \%)$ & $94(100 \%)$ & & & \\
\hline Hipertensão arterial sistêmica & & & & 0,51 & 1,21 & $0,71-2,04$ \\
\hline Sim & $97(32,6 \%)$ & $69(33,8 \%)$ & $28(29,8 \%)$ & & & \\
\hline Não & $201(67,4 \%)$ & $135(66,2 \%)$ & $66(70,2 \%)$ & & & \\
\hline Doenças respiratórias & & & & 0,27 & 1,63 & $0,74-3,58$ \\
\hline $\operatorname{Sim}$ & $39(13,1 \%)$ & $30(14,7 \%)$ & $9(9,6 \%)$ & & & \\
\hline Não & $259(86,9 \%)$ & $174(85,3 \%)$ & $85(90,4 \%)$ & & & \\
\hline Doenças renais & & & & 0,32 & 1,92 & $0,62-5,89$ \\
\hline Sim & $20(6,7 \%)$ & $16(7,8 \%)$ & $4(4,3 \%)$ & & & \\
\hline Não & $278(93,3 \%)$ & $188(92,2 \%)$ & $90(95,7 \%)$ & & & \\
\hline Doenças cardíacas & & & & 1,0 & 1,09 & $0,53-2,25$ \\
\hline Sim & $40(13,4 \%)$ & $28(13,7 \%)$ & $12(12,8 \%)$ & & & \\
\hline Não & $258(86,6 \%)$ & $176(86,3 \%)$ & $82(87,2 \%)$ & & & \\
\hline Doenças orais & & & & $0,02^{*}$ & 1,49 & $1,37-1,61$ \\
\hline Sim & $11(3,7 \%)$ & $11(5,4 \%)$ & 0 & & & \\
\hline Não & $287(96,3 \%)$ & $193(94,6 \%)$ & $94(100 \%)$ & & & \\
\hline Doenças cutâneas & & & & 0,80 & 1,31 & $0,46-3,75$ \\
\hline $\mathrm{Sim}$ & $19(6,4 \%)$ & $14(6,9 \%)$ & $5(5,3 \%)$ & & & \\
\hline Não & $279(93,6 \%)$ & $190(93,1 \%)$ & $89(94,7 \%)$ & & & \\
\hline Doenças oculares & & & & 0,50 & 1,34 & $0,67-2,66$ \\
\hline $\operatorname{Sim}$ & $49(16,4 \%)$ & $36(17,6 \%)$ & $13(13,8 \%)$ & & & \\
\hline Não & $249(83,6 \%)$ & $168(82,4 \%)$ & $81(86,2 \%)$ & & & \\
\hline Doenças vasculares & & & & 1,00 & 1,40 & $0,28-7,08$ \\
\hline Sim & $8(2,7 \%)$ & $6(3,0 \%)$ & $2(2,1 \%)$ & & & \\
\hline Não & $289(97,3 \%)$ & $197(97 \%)$ & $92(97,9 \%)$ & & & \\
\hline Doenças gastrintestinais & & & & 0,09 & 1,87 & $0,95-3,65$ \\
\hline Sim & $60(20,1 \%)$ & $47(23 \%)$ & $13(13,8 \%)$ & & & \\
\hline Não & $238(79,9 \%)$ & $157(77 \%)$ & $81(86,2 \%)$ & & & \\
\hline Doenças endócrinas & & & & 0,44 & 2,82 & $0,33-23,75$ \\
\hline Sim & $7(2,3 \%)$ & $6(2,9 \%)$ & $1(1,1 \%)$ & & & \\
\hline Não & $291(97,7 \%)$ & $198(97,1 \%)$ & $93(98,9 \%)$ & & & \\
\hline
\end{tabular}

Comparação entregrupos realizada por meio de teste qui-quadrado. *Associação significativa entre contato com agrotóxicose relato dedoença. 
Tabela 3. Análise da associação entre relato de doenças cardiovasculares, neurológicas e psiquiátricas pelos entrevistados e contato com agrotóxicos

\begin{tabular}{|c|c|c|c|c|c|c|}
\hline \multirow[b]{2}{*}{ Doenças relatadas } & \multirow[b]{2}{*}{ Frequências } & \multicolumn{2}{|c|}{ Contato com agrotóxicos } & \multirow[b]{2}{*}{$\mathrm{p}$} & \multirow{2}{*}{$\begin{array}{c}\text { Razão de } \\
\text { chances }\end{array}$} & \multirow[b]{2}{*}{ IC $95 \%$} \\
\hline & & $\operatorname{Sim}(n=204)$ & Não $(n=94)$ & & & \\
\hline Condições dolorosasa & & & & 0,02 & 1,93 & $1,13-3,30$ \\
\hline Sim & 109 & 84 & 25 & & & \\
\hline Não & 189 & 120 & 69 & & & \\
\hline Cardiovasculares ${ }^{\mathrm{b}}$ & & & & 0,20 & 1,52 & $0,83-2,78$ \\
\hline Sim & 241 & 169 & 72 & & & \\
\hline Não & 56 & 34 & 22 & & & \\
\hline Psiquiátricas ${ }^{c}$ & & & & 1,00 & 1,02 & $0,59-1,77$ \\
\hline Sim & 80 & 55 & 25 & & & \\
\hline Não & 218 & 149 & 69 & & & \\
\hline
\end{tabular}

Comparação entregrupos realizada por meio de teste qui-quadrado ou teste exato deFisher. al ncluiu o relato de cefaleia, neuropatia e artrite. 'Incluiu relato de hipertensão arterial sistêmica, doenças vasculares, cardíacas e renais, hiperglicemia, hipercolesterolemia, uso de tabaco e consumo de bebidas alcoólicas. 'Incluiu o relato de ansiedade, depressão, distúrbios desono.

asuscetibilidade dos tecidos a outros agentes químicos. 0 consumo de bebidas alcoólicas atua tanto de forma local (região oral), aumentando o risco de lesões orais quanto sistêmica conduzindo a desnutrição e depressão do sistema imunológico ${ }^{11}$. A diminuição da resposta imunológica favorece a ocorrência de neoplasias e infecções. Considerando estes dados, pode-se sugerir que o consumo do álcool por indivíduos com contato com agrotóxicos pode estar potencializando o risco de desenvolvimento de lesões orais relatadas por eles. Este fato justifica ter sido incluído o consumo de bebidas alcoólicas na análise multivariada, pois poderia estar sendo um fator confundidor em relação aos desfechos observados em indivíduos com contato com agrotóxicos, no entanto foi confirmada a associação entre contato com agrotóxicos e doenças orais assim como com neurológicas.

Foi constatada associação entre o contato com agrotóxicos e o relato de doenças neurológicas e também síndromes dolorosas. Indivíduos com contato com agrotóxicos apresentaram 2,5 vezes mais chances de relatar doenças neurológicas e 2 vezes mais chances de relatarem síndromes dolorosas do que os sem contato Agrotóxicos de vários grupos, como organofosforados, carbamatos, organoclorados, piretroides e outros, se associam a efeitos neurológicos agudos com exposições a altas doses. Pode haver sequelas tanto sensitivas quanto motoras, além de deficiências cognitivas transitórias ou permanentes. Cabe destaque à possível inter-relação entrea exposição crônica a agrotóxicos e o desenvolvimento de doenças degenerativas do sistema nervoso central ${ }^{12-14}$. Em estudo realizado em N ova Friburgo, Rio de Janei$\mathrm{ro}^{9}$, verificaram respostas alteradas ao exame neurológico periférico do sistema motor e sensitivo em uma amostra de 102 pequenos agricultores, de ambos os sexos, sugerindo neuropatia tóxica, com provável degeneração axonal ${ }^{15}$, destacam a possibilidade de associação entre exposição a agrotóxicos e doenças crônicas não-transmissíveis, tais como câncer, endocrinopatias, doenças neurológicas e outras. A prevalência de algumas dessas afecções tem se elevado, e contaminantes ambientais, como é o caso dos agrotóxicos, podem estar associados ao seu aparecimento, tendo, muitas vezes, origem multifatorial. Assim, éimportante o reconhecimento dessa possibilidade associativa pelo sistema de vigilância em saúde, que deve estar alerta para potenciais efeitos advindos de contaminações ambientais e ocupacionais e buscar pesquisar as causas ambientais, como os agrotóxicos, na gênese de agravos de natureza crônica.

0 presente trabalho não mostrou aumento de relato de câncer; porém, devido às limitações metodológicas, estudos adicionais devem ser de senvolvidos para melhor elucidar essa questão. Outros estudos epidemiológicos têm apontado para a possibilidade de associação entre vários tipos de câncer eexposição a agrotóxicos. Os efeitos crônicos dessa exposição, incluindo alterações neurológicas, reprodutivas e câncer ${ }^{16-19}$, são mais difíceis de determinar, mas há estudos que encontraram associação entre exposição a pesticidas e esses efeitos ${ }^{20-23}$. Intoxicações por agrotóxicos são frequentes entre os agricultores, determinando, por vezes, a proibição médica do 
trabalho na lavoura e a orientação para outro tipo de atividade profissional. Exposição ocupacional a pesticidas tem sido relacionada a efeitos prejudiciais agudos sobre a saúde, incluindo doenças que afetam pele, ol hos etrato respiratório, podendo levar à morte ${ }^{24,25}$.

Ao se avaliarem as doenças e as manifestações específicas relatadas pelos entrevistados, observou-se associação significativa entre o contato com agrotóxicos e o relato de doenças orais. Q uanto a estas últimas, nenhum dos indivíduos sem contato relatou a sua presença, enquanto $3,7 \%$ dos com contato referiram têlas. Sabe-se que efeitos colinérgicos agudos são frequentes nessas populações em decorrência da ação de substâncias inibidores da enzima acetilcolinesterase (AChE). A função da AChE é hidrolisar a acetilcolina ( $A C h$ ) liberada no sistema nervoso autônomo, no sistema nervoso central e na junção neuromuscular ${ }^{9}$. 0 efeito dessa inibição é 0 acúmulo de ACh nas respectivas fendas sinápticas, gerando efeitos periféricos e centrais, decorrentes da estimulação dos receptores muscarínicosenicotínicos ${ }^{9}$. Considerando que a exposição crônica a agrotóxicos induz a estimulação colinérgica decorrente da inibição da AChE, podendo gerar down-regulation de receptores muscarínicos com consequente decréscimo da atividade colinérgica, o que pode causar xerostomia, pode-se levantar a hipótese de que este seja um dos mecanismos envolvido no aumento de relato de lesões orais.

Por outro lado, houve significativa associação entre relato de doenças na família e exposição a agrotóxicos. Quanto ao número de doenças na família relatadas pelos entrevistados, também foram vista diferenças significativas entre indivíduos com e sem contato com agrotóxicos. Tais resultados confirmam que, em pequenas comunidades agrícolas, como é o caso da presente amostra, frequentemente a agricultura é uma atividade familiar, todos participam de alguma forma do processo de plantio, da adubagem, do combate às pragas e da colheita, tornando-os suscetíveis aos malefícios da exposição crônica a agrotóxicos.

$N$ ão houve diferença entre os grupos para relato de artrite quando se analisaram os dados pelo teste qui-quadrado, porém, quando os dados foram aplicados na regressão logística, houve correlação entre artrite e exposição a agrotóxicos. Vários estudos têm relatado aumento do risco de artrite reumatoide entre os trabalhadores rurais ${ }^{26-29}$. 0 uso de pesticidas tem sido associado com o aumento do risco de artrite reuma- toide (20\%-30\% de aumento) ${ }^{26-28}$. No entanto, pesticidas específicos não têm sido estudados epidemiologicamente. Relato de artrite reumatoide tem se mostrado subjetivo em outros estudos, com confirmação de menos de $21 \%-22 \%{ }^{30}$. ${ }^{31}$. Com casos frequentemente necessitando de validação por meio de registros médicos, os grupos para estudos epidemiológicos tendem a ser pequenos. De Roos e colaboradores ${ }^{32}$ encontraram modesta associação entre exposição a pesticidas e risco de artrite reumatoide, tanto para pesticidas em geral quanto para grupos específicos de pesticidas. Por outro lado, o estudo de Olsson e colaboradores ${ }^{29}$ sugeriram que o risco de artrite estava associado à ocupação rural, mas não ao relato de utilização de agrotóxicos, e dois outros trabal hos anteriores encontraram discreto aumento de risco (risco estimado de 1,2 e 1,3) associado ao uso de agrotóxicos ${ }^{27-28}$.

Os resultados aqui obtidos corroboram os de pesquisas anteriores edemonstram queéfundamental incentivar o conhecimento pormenorizado dos efeitos agudos e crônicos e que se estimulem de forma prioritária as restrições ao uso de agrotóxicos pelos riscos que oferecem. Deveseconsiderar também que muitos dos efeitos dos pesticidas permanecem no organismo por longo período mesmo após a suspensão da exposição ao agente. Esse efeito é decorrente da meia-vida longa, da existência de metabólitos ativos e de sua alta li possolubilidade que leva a depósito em tecido adiposo.

É de salientar que os resultados ora apresentados são decorrentes de um estudo transversal, o que torna importante a realização de estudos longitudinais para estabelecer a relação de causalidade. Quanto às limitações da forma deamostragem deve-se enfatizar que os sujeitos foram selecionados em um serviço de saúde, o que favorece o encontro de indivíduos com desfechos de interesse, porém pode aumentar a probabilidade de serem detectadas associações com o fator de estudo. Para uma próxima pesquisa, uma amostra representativa da população poderia ser obtida mediante amostragem em estágios múltiplos. Além disso, neste estudo não foram realizados exames clínicos ou toxicológicos adicionais, nem mesmo um levantamento sobre a inserção de cada respondente nos diversos processos produtivos que caracterizam a atividadeagrícola, portanto não se pode afirmar com certeza que a categoria denominada com contato apresente níveis toxicológicos deletérios à saúde. Como método de estudo, optou-se pelo método analítico, que permiteestudar associações defor- 
ma exploratória e possibilita levantar hipóteses, porém não propicia o aprofundamento das questões abordadas.

Concluindo, os dados deste trabalho corroboram os de outros estudos epidemiológicos que também demonstraram a necessidade de realização de projetos promotores de educação e de saúde entre a população em contato com agrotóxicos, além de fomento para pesquisas que visem estudos populacionais.

\section{Colaboradores}

A Souza, AR M edeiros, AC Souza foram os responsáveis pela coleta e digitação dos dados. IR Siqueira, M Wink, L Fernandes participaram da orientação dos alunos durante todo o período de coleta. M PL Hidalgo, M BC Ferreira realizaram análise estatística dos dados e confecção do artigo. IL Silva Torres elaborou e coordenou 0 projeto, orientou os alunos, participou da análise estatística dos dados e da confecção do artigo.

\section{Agradecimentos}

Às prefeituras dos municípios de Travesseiro, Westfália e Dr. Ricardo, pelo apoio dado às pesquisas. Pelo apoio financeiro: FAPERGS (Auxilio Recém-Doutor, BIC), CN Pq, Univates. 
1. Fairbanks M. Defensivos agrícolas ampliam o mercado. Rev Quím e Derivados 2001; 396:398-403.

2. Pires DX, Caldas ED, Recena M CP. Pesticide use and suicide in the State of Mato Grosso do Sul, Brazil. Cad Saude Publica 2005; 21(2):598-604.

3. Soares W, Almeida RM, Moro S. Trabalho rural e fatores de risco associados ao regime de uso de agrotóxicos em Minas Gerais, Brasil. Cad Saude Publica 2003; 19(4):1117-27.

4. Moreira JC, Jacob SC, Peres F. Avaliação integrada do impacto do uso de agrotóxicos sobre a saúde humana em uma comunidade agrícola de Nova Friburgo, RJ. Cienc Saude Coletiva 2002; 7(2):299-311.

5. Levigard, YE, Rozemberg B. A Interpretação dos Profissionais de Saúde acerca do N ervoso no M eio Rural [dissertação]. Rio de Janeiro (RJ): Centro de Estudos da Saúde do Trabalhador e Ecologia Humana. Escola Nacional de Saude Publica. Fundação Oswaldo Cruz; 2001.

6. Faria NMX, Facchini LA, Fassa AG, Tomasi E. Trabalho rural e intoxicações por agrotóxicos. Cad Saude Publica 2004; 20(5):1298-1308.

7. Schaumburg HH, Berger AR. "Human toxic neuropathy due to industrial agents". In: Dyck PJ, THOmas PK, organizadores. Peripheral Neuropathy. London: Saunders; 1993. p. 1533-1548.

8. Jamal GA. "Long-term neurotoxic effects of organophosphate compounds". Adverse Drug React Toxicol Rev 1995; 14(2):85-99.

9. Araújo AJ, de Lima JS, M oreira JC, Jacob SC, Soares MO, Monteiro MCM, do Amaral AM, Kubota A, Meyer A, Cosenza CAN, N eves C, Markowitz S. Exposição múltipla a agrotóxicos e efeitos à saúde: estudo transversal em amostra de 102 trabalhadores rurais N ova Friburgo, RJ. Cienc Saude Colet 2007; 12(1):115-130.

10. Ramirez $\mathrm{A}$. Análise de micronúcleos em alcoólicos portadores de carcinomas orais. [dissertação]. São Paulo (SP): Universidade de São Paulo; 2000.

11. Carrard VC, Pires AS, Paiva RL, Chaves ACM, Sant'Ana Filho M; Álcool e Câncer Bucal: Considerações sobre os M ecanismos Relacionados. Rev Bras de Cancerologia 2008; 54(1):49-56.

12. M eggs WJ. Permanent paralysis at sites of dermal exposure to chlorpyrifos. J Toxicol Clin Toxicol 2003; 41(6):883-886

13. Kamel F, Hoppin JA. Association of pesticide exposure with neurologic dysfunction and disease. Environ Health Persp 2004; 112(9):950-958.

14. Lotti M, Moretto A. Organophosphate-induced delayed polyneuropathy. Toxicol Review 2005; 24(1): 37-49.

15. Miranda AC, Moreira JC, Carvalho R, Peres F. Neoliberalismo. Uso de agrotóxicos e a crise da soberania alimentar no Brasil. Cienc Saude Colet 2007; 12(1):7-14.

16. Fong R, M einders D. Oxydemeton-methyl (metasystox-r): M etabolic fate dermal transport and human exposure data. HS-1536. Sacramento, California: Department of Food and Agriculture; 1990.

17. Fong R. Bromoxynil: metabolic fate, dermal transport and human exposure data (appendix B). HS-1467. Sacramento, California: Department of Food and Agriculture; 1991.
18. Formoli A, Ross H. Estimation of exposure of persons in California to pesticide products that contain paraquat. HS-1560. Sacramento, California: Department of Food and Agriculture; 1991.

19. Formoli A. Estimation of exposure of persons in California to the pesticide products that contain diquat dibromide. HS-1662. Sacramento, California: Environmental Protection Agency; 1993.

20. Sharp DS, Eskenazi B, Harrison R, Callas P, Smith $A H$. Delayed health hazards of pesticide exposure. Annu Rev Public Health 1986; 7:441-471.

21. Blair A, Zahm S, Pearce NE, H eineman EE, Fraumeni JF. Clues to cancer etiology from studies of farmers. Scand J Work Environ Health 1992; 18(4):209-215.

22. Davis D, Blair A, Hoel D. Agricultural exposures and cancer trends in developed countries. Environ Health Perspect 1992; 100:39-44.

23. Blair A, Dosemeci M, Heineman EF. Cancer and other causes of death among male and female farmers from twenty-three states. Am J Ind M ed 1993; 23(5):729-742.

24. Maddy K, Edmiston S, Richmond D. Illness, injuries, and deaths from pesticide exposures in California 1949-1988. Rev Environ Contam Toxicol 1990; 114:57-123.

25. Mehler $L, O^{\prime} M$ alley MA, Krieger RI. Acute pesticide morbidity and mortality: California. Rev Environ Contam Toxicol 1992; 129:51-66.

26. Milham S. Using multiple cause of death coding in occupational mortality studies. Am J Ind Me. 1988; 14(3):341-344.

27. Lundberg I, Alfredsson L, Plato N, Sverdrup B, Klareskog L, Kleinau S. Occupation, occupational exposure to chemicals and rheumatological disease: A register based cohort study. Scand J Rheumatol 1994; 23(6):305-310.

28. Olsson AR, Skogh T, Wingren G. Occupational determinants for rheumatoid arthritis. Scand J Work Environ Health 2000; 26(3):243-249.

29. Olsson AR, Skogh T, Axelson O, Wingren G. Occupations and exposures in the work environment as determinants for rheumatoid arthritis. Occup Environ M ed 2004; 61(3):233-238.

30. Kvien TK, Glennas A, Knudsrod OG, Smedstad LM. The validity of self-reported diagnosis of rheumatoid arthritis: Results from a population survey followed by clinical examinations. J Rheumatol 1996; 23(11):1866-1871.

31. Star VL, Scott JC, Sherwin R, Lane N, Nevitt MC, Hochberg MC. Validity of self-reported rheumatoid arthritis in elderly women. J Rheumatol 1996; 23(11):1862-1865.

32. De Roos AJ, Cooper GS, Alavanja MC, Sandler DP. Rheumatoid arthritis among women in the agricultural health study: risk associated with farming activities and exposures. Ann Epidemiol 2005; 15(10): 762-770.

Artigo apresentado em 13/10/2007

Aprovado em 27/07/2008

Versão final apresentada em 03/08/2008 